\title{
Präklinische Studie:
}

\section{Probiotika als neue Therapie- option bei intestinaler} Fruktoseintoleranz

\author{
Kati Goroncy
}

Die Aufnahme von Fruktose mit der Nahrung kann bei Menschen mit intestinaler Fruktoseintoleranz (IFI) Beschwerden wie Durchfall und Blähungen auslösen. Mit zunehmendem Fruktosegehalt in der Nahrung sind immer mehr Menschen betroffen. Der Artikel beschreibt Ursachen und Symptome einer IFI und stellt anhand jüngster präklinischer Studien dar, wie sie mithilfe probiotischer Bakterien vermieden werden könnten.

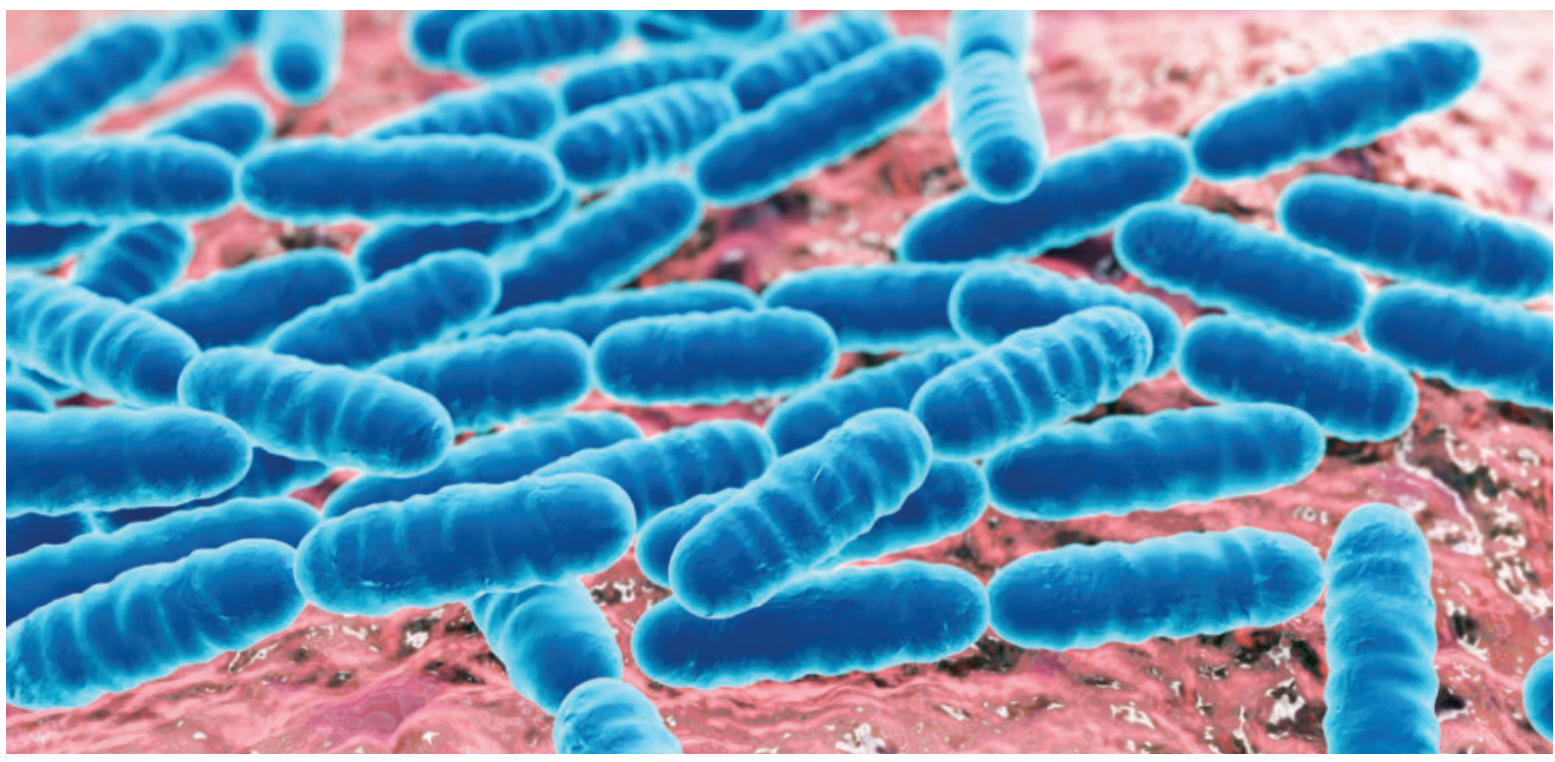

Nützliche Helfer: Lactobacillus-Arten gehören zu den Probiotika. Sie bauen Fruktose in vitro effektiv ab und könnten im Dünndarm vielleicht auch die Fruktoseintoleranz lindern. Foto: Adobe Stock/Kateryna_Kon

\section{Fruktose in der Nahrung}

Die umgangssprachlich als Fruchtzucker bekannte Fruktose ist ein Monosaccharid, das beispielsweise in Obst, Trockenobst, Fruchtsäften und Honig vorkommt. Zusammen mit Glukose ist Fruktose Bestandteil des Disaccharids Saccharose, dem sogenannten Haushaltszucker. Fruktose wird mittlerweile in vielen industriell produzierten Lebensmitteln, aber auch in Light-Produkten und Diabetikerprodukten als Süßungsmittel eingesetzt [1], da Fruktose als süßer als Glukose empfunden wird und dabei einen gerin- geren Anteil an Kalorien aufweist. Der Fruktosekonsum hat in den letzten Jahrzehnten aufgrund der extensiven Nutzung des reinen Monosaccharids und des aus Mais gewonnenen Fruktose-Glukose-Sirups (HFCS = high-fructose corn syrup, Isoglukose) dramatisch zugenommen [2].

Merke

Im Oktober 2017 ist die europäische Quotenregelung für Zucker abgelaufen. Deshalb könnte HFCS bald auch verstärkt auf dem europäischen Markt Einzug halten. 
Besonders beliebt ist der HFCS-Zusatz bei Erfrischungsgetränken, Säften und Honig. So finden sich zum Beispiel in einem Liter mit HFCS gezuckerten Fruchtsäften bis zu $65 \mathrm{~g}$ Fruktose [3]. Diese vermehrte Fruktoseaufnahme von weltweit durchschnittlich 11-54g pro Person und Tag [4] bei normaler Ernährung wird bereits als Risikofaktor für die Entstehung von Übergewicht und dem metabolischen Syndrom diskutiert [1][5].

\section{Störung der Fruktoseaufnahme}

Etwa ein Drittel der Menschen zeigt schon bei einer Aufnahme von $25 \mathrm{~g}$ Fruktose eine gestörte Resorption im Dünndarm, eine sogenannte Fruktosemalabsorption [6]. Grund hierfür ist eine dosisabhängige Überlastung des Fruktose-Transporters GLUT5 [7], wodurch der Körper die vorliegende Fruktose nicht vollständig über den Dünndarm aufnehmen kann. Die nicht resorbierte Fruktose gelangt so in den Dickdarm, wo sie von Bakterien hauptsächlich zu Wasserstoff, Kohlendioxid und zu kurzkettigen Fettsäuren verstoffwechselt wird [8].

\section{Intestinale Fruktoseintoleranz}

\section{Symptome}

Bei der Hälfte der Personen mit Fruktosemalabsorption führen die im Dickdarm entstandenen Gärprodukte zu Symptomen einer intestinalen Fruktoseintoleranz (IFI) [9]. IFI ist gekennzeichnet durch klinische Symptome wie Blähungen, Bauchschmerzen und Durchfall. Gehäuft treten Zeichen von Müdigkeit, Depression und ein Vitaminund Spurenelementmangel auf [10][11]. Bei chronischer Fruktosemalabsorption kann es zu einer bakteriellen Fehlbesiedlung des Dünndarms und einem Reizdarmsyndrom kommen [12].

\section{DIFFERENZIALDIAGNOSE HFI}

Klar abzugrenzen von der IFI ist die hereditäre Fruktoseintoleranz (HFI). Bei dieser sehr seltenen, erblich bedingten Krankheit fehlt das Leberenzym Aldolase B. Die bisher einzige Therapie für Betroffene ist eine lebenslange fruktosefreie Diät.

\section{Probiotika als möglicher Therapieansatz bei IFI?}

Aktuelle Therapieempfehlungen bei IFI beschränken sich auf eine fruktosearme Diät und die Aufnahme des Enzyms Xylose-Isomerase. Eine fruktosearme Ernährung ist nicht immer ganz einfach und die zugeführte Enzymaktivität ist häufig zu niedrig, um effektiv Fruktose abzubauen.

Milchsäurebakterien sind bekannt für ihre probiotische Wirkung und für die Aufrechterhaltung einer gesunden Darmflora, weshalb sie bereits vielfach in der Therapie eingesetzt werden:

- zur Linderung von Darmentzündungen, 


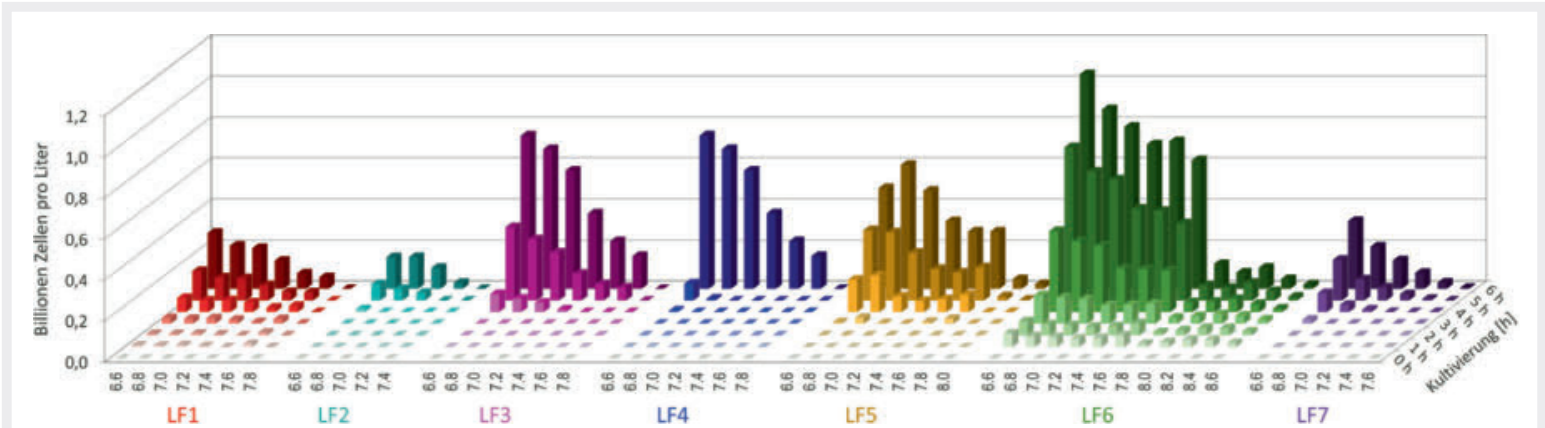

Abb. 1 pH-Wachstumstest aller untersuchten Stämme von L. fermentum. Ein „darmähnliches“ Medium wurde auf einen Anfangs-pH-Wert von 6,6-8,6 eingestellt (X-Achse). Die Kulturen wurden bei $37^{\circ} \mathrm{C}$ für $6 \mathrm{~h}$ inkubiert (Z-Achse) und anschließend wurde die Lebendzellzahl bestimmt (Y-Achse).

- zur Normalisierung bei Darmschleimhaut-Barrierestörungen,

- zur Herabregulierung von Überempfindlichkeitsreaktionen und

- zur Stärkung des Immunsystems [13][14].

Ein neuer Therapieansatz bei IFI könnte die Verwendung probiotischer Milchsäurebakterien sein, die Fruktose im Dünndarm aufnehmen und verstoffwechseln, bevor das Monosaccharid im Dickdarm Symptome auslöst. Im Zuge der hier vorgestellten präklinischen Studie sollten verschiedene Bakterienstämme in vitro auf ihre Wirkung und Unbedenklichkeit untersucht werden.

\section{In-vitro-Studie: Gesucht...}

\section{Bakterium im Dünndarm...}

Im Labor wurden zunächst diverse probiotische Bakterienstämme, darunter Bifidobacterium breve, Bifidobacterium infantis, Bifidobacterium lactis, Lactobacillus delbrueckii ssp. bulgaricus und Lactobacillus fermentum auf die Verstoffwechselung von Fruktose untersucht. Der vielversprechendste Stamm Lactobacillus fermentum (LF) wurde eingehender auf seine Eignung als Mittel gegen IFI analysiert.

Um zu verhindern, dass überschüssige Fruktose in den Dickdarm gelangt, sollte der ideale Stamm im neutralen bis leicht basischen Dünndarmmilieu mit pH-Werten zwischen 6,6 und 8,6 [15] überleben können, um die Fruktose aufzunehmen.

Ergebnis: Lactobacillus-fermentum-Stämme von 7 verschiedenen Anbietern (LF1-7) zeigen unterschiedliche Wachstumsprofile bei $37^{\circ} \mathrm{C}$ über $6 \mathrm{~h}$ ( $\mathbf{A b b}$. 1). Allen Stämmen gemein ist eine Latenzphase von mehreren Stunden sowie die Präferenz saurer pH-Werte. Der Stamm LF6 weist das stärkste Wachstum auf.

\section{... mit Vorliebe für Fruktose}

Der mögliche Einsatz von Lactobacillus fermentum zur Prävention und Therapie von IFI erfordert nicht nur ausrei- chendes Wachstum, um das Überleben des Stammes zu sichern, sondern ebenso die Verwertung von Fruktose. Was einfach klingen mag, ist nicht selbstverständlich: Fruktose wird selten in reiner Form aufgenommen. Typische Mahlzeiten enthalten auch Glukose sowie Saccharose, die im Darm zu Fruktose und Glukose aufgespalten wird. Der ideale Stamm verwertet Fruktose daher auch in Gegenwart von Glukose, insbesondere auch, weil Wachstum mit Glukose als Energiequelle im Zuge der Milchsäuregärung häufig zu Ethanol abgebaut wird, dem handelsüblichen Alkohol. Im Labor wurde eine Kapselmenge mit getrocknetem, inaktiven Lactobacillus fermentum versetzt mit

- (I.) reiner Glukose, wie z. B. in Traubenzucker-Bonbons,

- (II.) reiner Fruktose, wie z. B. in Säften oder Beeren, und

- (III.) einer Mischung aus beiden Zuckern wie bei einer natürlichen Mahlzeit.

Dabei wurden das Wachstum, die Fruktoseverwertung sowie die Bildung von Ethanol gemessen (- Abb. 2).

Ergebnis: LF4 bevorzugt Glukose, denn der Stamm wächst deutlich schneller auf Glukose und verwertet aus dem gemischten Medium nur sehr wenig Fruktose. LF4 bildet zudem bis zu $20 \mathrm{~g}$ Ethanol auf reiner Glukose und gemischtem Medium

LF6 und LF7 hingegen bevorzugen Fruktose als Energiequelle, sie wachsen auf Fruktose schneller und verbrauchen viel Fruktose (19,8 g und 12,1 g). Das Wachstum auf reiner Glukose ist hingegen gehemmt und selbst bei Wachstum auf der Fruktose-Glukose-Mischung werden kaum messbare Mengen an Ethanol gebildet. LF6 zeigte das schnellste Wachstum und den höchsten Fruktoseverbrauch; der Stamm konnte ca. $50 \%$ der Fruktose in $9 \mathrm{~h}$ verwerten. LF7 zeichnete sich durch die niedrigste Menge an gebildetem Ethanol aus: 3,5 g Ethanol bei Verstoffwechselung von $60 \mathrm{~g} / \mathrm{l}$ Glukose. 

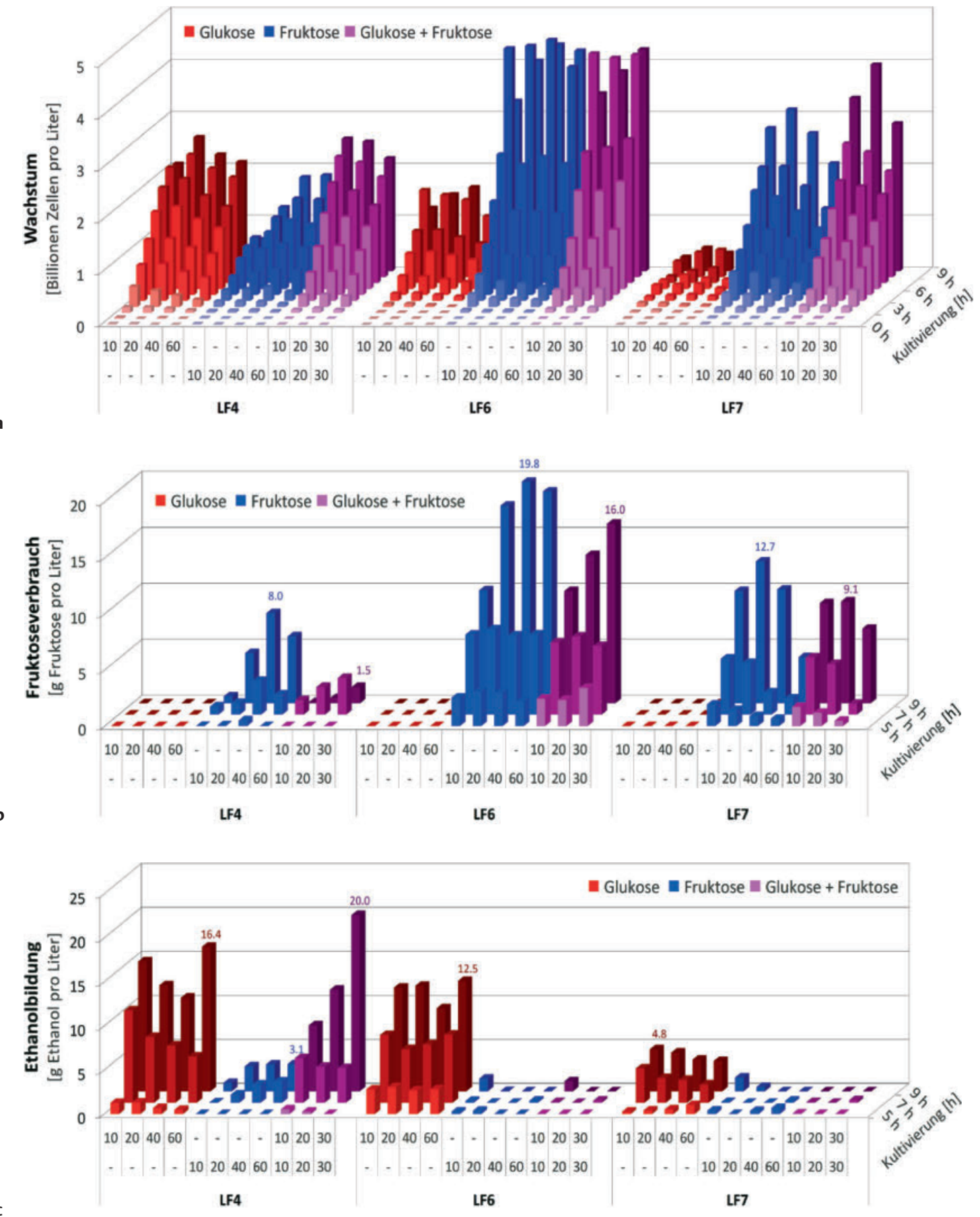

- Abb. 2 a Wachstum, b Fruktoseverbrauch und c Ethanolbildung der Stämme LF4, LF6 und LF7 in einem Minimalmedium mit

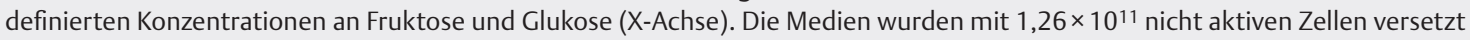
und bei $37^{\circ} \mathrm{C}$ bis zu 9 Stunden bei einem anfänglichem pH-Wert von 6 anaerob kultiviert (Z-Achse). Jede Stunde wurde die Anzahl gewachsener Zellen (a; Y-Achse), der Verbrauch von Fruktose (b; Y-Achse) und die Ethanolbildung (c; Y-Achse) bestimmt. In rot ist das Wachstum, der Fruktoseverbrauch und die Ethanolbildung auf reiner Glukose, in blau auf reiner Fruktose und in magenta auf einer Mischung der beiden Zucker dargestellt. 


\section{BEMERKUNG}

Das in Spuren gebildete Ethanol wird in vivo erwartungsgemäß niedriger ausfallen als in vitro, da die hierfür erforderliche Glukose schnell über den Darm aufgenommen sowie vom übrigen Mikrobiom verstoffwechselt wird.

\section{Anwendungsempfehlungen}

Aufbauend auf den präklinischen Daten ergeben sich folgende Empfehlungen bezüglich der Anwendung in einer folgenden klinischen Studie. Aufgrund der mehrstündigen Latenzphase ist eine regelmäßige, tägliche Einnahme empfohlen. Um eine hohe Inokulationsmenge zu gewährleisten, ist die Darreichung in magensäureresistenten Kapseln angeraten. Bei einer Lebendzellzahl (CFU) von ca. 1,3 Milliarden LF7-Zellen konnten in $9 \mathrm{~h}$ unter Laborbedingungen 12,5 gruktose verwertet werden. In der Folge könnte die tägliche Einnahme einer Kapsel mit einer Lebendzellzahl von 5 Milliarden LF7 genügen, um überschüssige Fruktose zu entfernen und Symptomen der intestinalen Fruktoseintoleranz entgegenzuwirken. In der Karenzphase kann die Erhöhung auf 2 Kapseln sinnvoll sein, um die Fruktose vollständig aus dem Dünndarm zu entfernen. Die zusätzliche Einnahme von Präbiotika mit Fructooligosacchariden wie Inulin kann die Wirksamkeit reduzieren. Durch die Mischung des Stammes LF7 mit einem Stamm mit unterschiedlichem pH-Profil kann eine breitere Ansiedlung im Dünndarm erzielt werden, um den Fruktoseumsatz bei Bedarf weiter zu erhöhen.

\section{Vorsicht}

LF7 ist nicht zur Anwendung bei hereditärer Fruktoseintoleranz geeignet.

\section{KERNAUSSAGEN}

Erste Schritte zur Entwicklung eines innovativen Therapieansatzes bei IFI

Die Einnahme von probiotischen Stämmen stellt einen innovativen Therapieansatz zur Behandlung von intestinaler Fruktoseintoleranz dar. In der präklinischen Studie wurden 7 verschiedene Stämme von Lactobacillus fermentum in vitro auf ihre Wirksamkeit und Unbedenklichkeit für den Einsatz in einer klinischen Studie getestet. Der Lactobacillusfermentum-Stamm LF7 zeigt hohes Potenzial für eine Anwendung bei intestinaler Fruktoseintoleranz aufgrund seiner selektiven Aufnahme von Fruktose und der damit verbundenen geringen Ethanolbildung. LF7 kann nun unbedenklich in eine klinische Studie mit Probanden eingesetzt werden, um die therapeutische Wirksamkeit zu untersuchen. Erste $\mathrm{H}_{2}$-Atemtest-Ergebnisse sehen vielversprechend aus.

\section{Interessenkonflikt}

Die Autorin erklärt, dass keine Interessenkonflikte zu Probiotikaherstellern bestehen, deren Produkte in den dargelegten Forschungen untersucht wurden. Die Forschungen zur Nutzung der Probiotika wurden von der AixSwiss B.V. unterstützt.

Über die Autorin

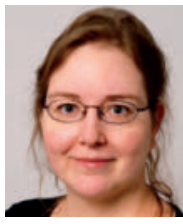

\section{Kati Goroncy}

ist Diplom-Biologin mit Schwerpunkt auf Mikrobiologie und Enzymologie. Nach mehrjähriger Tätigkeit in der Forschung an der Universität RWTH Aachen im Bereich Pflanzenbiotechnologie und bakterieller Zellkultur ist sie seit 2013 leitende Wissenschaftlerin bei dem Biotechnologieunternehmen SeSaM-Biotech GmbH.

Korrespondenzadresse

Dipl.-Biol. Kati Goroncy

SeSaM-Biotech GmbH

Forckenbeckstraße 50

52074 Aachen

E-Mail: goroncy@sesam-biotech.com

Literatur

Die Literatur ist in der Online-Version unter www.thieme-connect.de/products verfügbar.

Bibliografie

DOI https://doi.org/10.1055/a-0831-6177

Ernährung \& Medizin 2019; 34: 122-126

(c) Georg Thieme Verlag KG Stuttgart · New York ISSN 1439-1635 